BULL. AUSTRAL. MATH. SOC.

\title{
COMMUTATOR OF TWO PROJECTIONS \\ IN PREDICTION THEORY
}

\author{
TAKaHI KO NAKAZI
}

Let $w$ be a nonnegative weight function in $L^{1}=L^{1}(d \theta / 2 \pi)$. Let 2 and $P$ denote the orthogonal projections to the closed linear spans in $L^{2}(\omega d \theta / 2 \pi)$ of $\left\{e^{i n \theta}: n \leqslant 0\right\}$ and $\left\{e^{i n \theta}: n>0\right\}$, respectively. The commutator of $Q$ and $P$ is studied. This has applications for prediction problems when such a weight arises as the spectral density of a discrete weakly stationary Gaussian stochastic process.

\section{Introduction}

Let $w$ be a nonnegative weight function in $L^{1}=L^{1}(d \theta / 2 \pi)$.

Let $Z^{-}$denote the closed linear span in $Z$ of $\left\{e^{i n \theta}: n \leqslant 0\right\}$ and $Z^{+}$ denote the closed linear span in 2 of $\left\{e^{i n \theta}: n>0\right\}$, where $Z=L^{2}(\omega d \theta / 2 \pi)$. 2 denotes the orthogonal projection onto $Z^{-}$in $Z$ and $P$ denotes the orthogonal projection onto $Z^{+}$in $Z$. In this paper we assume that $\log w \in L^{1}$ because $Z=Z^{-}=Z^{+}$in the case that $\log w \notin L^{1}$. Hence we may assume $w=|h|^{2}$ for some outer function $h$ in $H^{2}$, the Hardy space for the unit disc. Then $z^{-}=\overline{h^{-1}} \bar{H}^{2}$ and $z^{+}=h^{-1} z H^{2}$. Set

$$
\phi=\hbar / h \text {. }
$$

Let $P$ be the orthogonal projection from $L^{2}$ onto $H^{2}$ and $Q=I-P$ where $I$ is the identity operator on $L^{2}$. Let $P_{0}$ be the

Received 24 September 1985. The author would like to thank Professor K. Takahashi for his helpful discussions. This research was partially supported by Grant-in-Aid for Scientific Research, Ministry of Education.

Copyright Clearance Centre, Inc. Serial-fee code: 0004-9727/86 $\$ A 2.00+0.00$. 
orthogonal projection from $L^{2}$ onto $H^{2} \cap \bar{H}^{2}$. Put $M_{k} f=k f, k \in L^{\infty}$ and $f \in L^{2}$. Then $Q+P_{0}=M_{z} Q M_{z}$ and $P-P_{0}=M_{z} P M_{z}$. Let $H_{k}$ denote the Hankel operator on $H^{2}$ defined by $H_{k} f=Q\left(M_{k} f\right)$, and $T_{k}$ denote the Toeplitz operator on $H^{2}$ defined by $T_{k} f=P\left(M_{k} f\right)$.

In this paper it is shown that $\|Q P-P Q\| \leqslant 1 / 4$. Let $H_{\phi}^{*} H_{\phi}=\int_{0}^{1} \lambda d E_{\lambda}$. Then it is shown that $Q P-P Q$ is compact if and only if $\int_{0}^{\varepsilon} \lambda d E_{\lambda}$ is compact for any $\varepsilon$ with $0<\varepsilon<1$. Also $Q P-P Q$ is compact and $Z^{-}+Z^{+}=Z$ if and only if $H_{\bar{\phi}}$ is compact. It is show that $H_{\bar{\phi}}$ is compact if and only if $\int_{0}^{1} \lambda d E{ }_{\lambda}$ is compact.

Helson and Sarason [2] showed that $Q P$ is compact if and only if $H_{\phi}$ is compact. Levinson and McKean ([4], pp. 103-105) studied the weight functions which satisfy $Q P-P Q=0$ and Hayashi [1] considered the operator $Q P-Q \wedge P$ where $Q \wedge P$ denote the orthogonal projection onto $Z^{-} \cap Z^{+}$in $Z$.

The problem of characterizing the weight functions $w$ that satisfy some kind of interdependence of $Z^{-}$and $Z^{+}$is of interest in the theories of weighted trigonometric approximations and discrete weakly stationary Gaussian stochastic process (such weights arise as the spectral densities of processes). We call $Z^{-}$the past of $Z$ and $Z^{+}$the future of $Z$. The results in this paper (see [1], [2], [3] and [5]) have applications for prediction problems when such a weight arises as the spectral density of a discrete weakly stationary Gaussian stochastic process.

\section{Norm of $Q P-P Q$}

We give three lemmas which relate the commutator $Q P-P Q$ and the Hankel operator of $\phi$ where $\phi=\hbar / h$ and $w=|h|^{2}$.

LEMMA 1. For $k \in L^{2}, \overline{Q h}^{-1} k=\bar{h}^{-1} M_{z} Q M-k$ and $P h^{-1} k=h^{-I_{M}}{ }_{z} M_{z} k$.

Proof. Since $Z=\overline{h^{-1}} L^{2}=\overline{h^{-1}} \bar{H}^{2} \oplus \overline{h^{-1}} z H^{2}, \overline{h^{-1} k}=\overline{h^{-1}}\left(Q+P_{0}\right) k=$ 
$\bar{h}^{-1} M_{z} Q M-k, k \in L^{2}$. The statement for $P$ follows similarly.

LEMMA 2. Let $k \in L^{2}$. Then the following hold :

(1) $Q P h^{-1} k=\bar{h}^{-1} M_{z} Q M_{\phi} P M_{z}^{-k}$,

(2) $(Q P-P Q) h^{-1} k=h^{-1} M_{z}\left(M_{\bar{\phi}} Q M_{\phi} P-P M_{\bar{\phi}} Q M_{\phi}\right) M_{z}^{-k}$.

Proof. This is clear.

LEMMA 3. Let $A=M_{\phi} Q M_{\phi} P-P M_{\phi} Q M_{\phi}$. Then $A \mid H^{2}=-H_{\phi} T_{\phi}$ and $A \mid \bar{z} \bar{H}^{2}=T_{\bar{\phi}} H \frac{*}{\phi}$.

Proof. We shall prove only $A \mid H^{2}=-H_{\phi} T_{\phi}$, since $A \mid \bar{z} \bar{H}^{2}=T_{\bar{\phi}} H_{\bar{\phi}}$ follows similarly.

$$
\begin{aligned}
A \mid H^{2} & =\left(M_{\phi} Q M_{\phi} P-P M_{\phi} Q M_{\phi} P\right) \mid H^{2} \\
& =Q M_{\phi} Q M_{\phi} P \mid H^{2} \\
& =Q M_{\phi}(I-P) M_{\phi} P \mid H^{2} \\
& =-H_{\phi} T_{\phi} .
\end{aligned}
$$

The map $S: f \rightarrow h f$ is an isometry of $Z$ onto $L^{2}$. Then

$$
M_{z} S(Q P-P Q)=\left(M_{\bar{\phi}} Q M_{\phi} P-P M_{\phi} Q M_{\phi}\right) M_{z} S
$$

THEOREM 1. $\|Q P-P Q\| \leqslant 1 / 4$.

Proof. By the remark above, $\|Q P-P Q\|=\left\|H_{\phi} T_{\phi}\right\|$.

$$
\begin{aligned}
\left(H_{\phi} T_{\phi}\right){ }^{*} H_{\phi} T_{\phi} & =T_{\bar{\phi}}\left(I-T_{\phi} T_{\bar{\phi}}\right) T_{\phi}=T_{\bar{\phi}} T_{\phi}\left(I-T_{\bar{\phi}} T_{\phi}\right) \\
& =\left(I-H_{\phi}^{*} H_{\phi}\right) H_{\phi}^{*} H_{\phi} .
\end{aligned}
$$

If $H_{\phi}^{*} H_{\phi}=\int_{0}^{1} \lambda d E{ }_{\lambda}$ then

$$
\left(H_{\bar{\phi}} T_{\phi}\right)^{*} H_{\bar{\phi}} T_{\phi}=\int_{0}^{1} \lambda(1-\lambda) d E_{\lambda}
$$

and this implies $\|Q P-P Q\| 1 / 4$. 
A theorem of Helson and Szegö [3] shows that $\|Q P\|<1$ if and only if $\sigma\left(H_{\phi}^{*} H_{\phi}\right) \subset[0,1-\varepsilon]$ for some $\varepsilon>0$ where $\sigma\left(H_{\phi}^{*} H_{\phi}\right)$ is the spectrum of $H_{\phi}^{*} H_{\phi}$. The proof of Theorem 1 shows that $\|Q P-P Q\|<1 / 4$ if and only if $\sigma\left(B_{\phi}^{*} H_{\phi}\right) \subset[0,1 / 2-\varepsilon] \cup[1 / 2+\varepsilon, 1]$.

\section{Compactness of $Q P-P Q$}

$w=|h|^{2}$ for some outer function $h$ in $H^{2}$ and $\phi=\hbar / h$. Let

$$
H_{\phi}^{*} H_{\phi}=\int_{0}^{1} \lambda d E_{\lambda}
$$

THEOREM 2. The following three properties are equivalent.

(1) $Q P-P Q$ is compact;

(2) $H_{\phi} T_{\phi}$ is compact ;

(3) For any $\varepsilon$ with $0<\varepsilon<1, \int_{0}^{\varepsilon} \lambda d E_{\lambda}$ and $\int_{\varepsilon}^{1}(1-\lambda) d E_{\lambda}$ are compact.

Proof. Lemmas 2 and 3 imply (1) $\Leftrightarrow$ (2).

(2) $\Leftrightarrow$ (3). $\left(H_{\phi} T_{\phi}\right){ }^{*} H_{\phi} T_{\phi}=\int_{0}^{1} \lambda(1-\lambda) d E_{\lambda}$ by the proof of Theorem 1 .

Hence $H_{\phi} T_{\phi}$ is compact if and only if for any $\varepsilon$, with $0<\varepsilon<1$, $\int_{0}^{\varepsilon} \lambda(1-\lambda) d E_{\lambda}$ and $\int_{\varepsilon}^{1} \lambda(1-\lambda) d E_{\lambda}$ are compact, that is if and only if

holds.

THEOREM 3. The following three properties are equivalent.

(1) $Q P-P Q$ is compact and $2^{-}+Z^{+}=2$;

(2) $H_{\bar{\phi}}$ is compact;

(3) For any $\varepsilon$, with $0<\varepsilon<1, \int_{0}^{\varepsilon} \lambda d E_{\lambda}$ is compact and $\int_{\varepsilon}^{1}(1-\lambda) d E_{\lambda}$ has finite rank.

Proof. (1) $\Rightarrow$ (2). Since $\overline{h^{-1}} \bar{H}^{2}+h^{-1} z H^{2}=h^{-1} L^{2}, \bar{z} \bar{H}^{2}+\phi H^{2}=L^{2}$ and $T_{\phi}$ is right invertible. By Theorem $2, H_{\bar{\phi}}$ is compact.

(2) $\Rightarrow$ (3). If $H_{\bar{\phi}}$ is compact and $\| H_{\bar{\phi}} \mathbb{\|}=1$ then there exists $f$ 
in $H^{2}$ with $\|f\|_{2}=1$ such that $H \frac{\star}{\phi} H \frac{f}{\phi}=f$ and so $T_{\bar{\phi}} f=0$. This contradicts $\operatorname{ker} T_{\bar{\phi}}=\{0\}$. Hence if $H_{\bar{\phi}}$ is compact then $\left\|H_{\bar{\phi}}\right\|<1$. Thus $T_{\phi} H^{2}=H^{2}$ and so $\left\|T_{\phi} f\right\|_{2} \geqslant \delta\|f\|_{2}, f \in M$ for some $\delta>0$ where $M=\left(\operatorname{ker} T_{\phi}\right)^{\perp}$. Let $P_{M}$ be the orthogonal projection from $L^{2}$ onto $M$. Then there exists a bounded linear operator $K$ such that $K T_{\bar{\phi}} T_{\phi}=P_{M}$. $P_{M} H_{\phi}^{*} H_{\phi}$ is compact because $H_{\bar{\phi}}$ is compact and $\left(H_{-} T_{\phi}\right)^{*} H_{\phi} T_{\phi}=T_{\phi} T_{\phi} H_{\phi}^{*} H_{\phi}$. Since $\operatorname{ker} T_{\phi}=\left(E_{1}-E_{1-0}\right) H^{2}, P_{M}^{H_{\phi}^{*} H_{\phi}}=H_{\phi}^{*} H_{\phi} P_{M}$. Thus $H_{\phi}^{*} H_{\phi} P_{M}$ is compact and so $\int_{0}^{1-} \lambda d E \lambda$ is compact. This implies (3).

(3) $\Rightarrow$ (2). By Theorem $2 H_{\phi} T_{\phi}$ is compact. Since $\int_{0}^{1-} \lambda d E_{\lambda}$ is compact, $\left\|T_{\phi} f\right\|_{2} \geqslant \delta\left\|^{\|}\right\|_{2}, f \in M$ for some $\delta>0$ because $H_{\phi}^{*} H_{\phi}\left|M=\left(I-T_{\phi}^{*} T_{\phi}\right)\right| M$ is compact. This implies that $T_{\phi}$ is right invertible and so $H_{\bar{\phi}}$ is compact.

(2) $\Rightarrow$ (1). As in the proof of (2) $\Rightarrow$ (3) if $H_{\bar{\phi}}$ is compact then $T_{\phi}$ is left invertible. $T_{\phi}$ is left invertible if and only if $\bar{z} \bar{H}^{2}+\phi H^{2}=L^{2}$. This and Theorem 2 imply (1).

THEOREM 4. The following three conditions are equivalent.

(1) $Q P-P Q$ has finite ronk $2 n$;

(2) $H_{\bar{\phi}}$ has finite rank $n$;

(3) for any $\varepsilon$, with $0<\varepsilon<1, \int_{0}^{\varepsilon} \lambda d E_{\lambda}$ has finite rank $m$ and $\int_{\varepsilon}^{1}(1-\lambda) d E_{\lambda}$ has finite rank $\ell$ with $m+\ell=n$.

Proof. By Lemma 3 rank $(Q P-P Q)=2$ rank $H_{\phi} T_{\phi}$. Since $\operatorname{ker} T_{\bar{\phi}}=\{0\}$,

$\operatorname{rank} H_{\bar{\phi}} T_{\phi}=\operatorname{rank} T_{\bar{\phi}} H \frac{*}{\phi}=\operatorname{rank} H \frac{*}{\phi}=\operatorname{rank} H_{\bar{\phi}}$

These imply (1) $\Leftrightarrow$ (2). (2) $\Leftrightarrow$ (3) can be shown in a similar manner to $(2) \Rightarrow(3)$ in Theorem 3 . 


\section{Weight functions}

$w=|h|^{2}$ for some outer function $h$ in $H^{2}$ and $\phi=\hbar / h$. Let $C$ denote the set of all continuous functions on the unit circle. Using a theorem of wolff [6] we can show that $w=\left|h_{0}\right|^{2} e^{u+v}$ where $h_{0}$ is an outer function in $H^{2-}=\underset{p<2}{n} H^{p}, \bar{h}_{0} / h_{0}=\bar{F} G$ for some inner functions $F$ and $G$ and where $u$ and $v$ are real functions in $C(\tilde{v}$ denotes the harmonic conjugate of $v$ ).

PROPOSITION 5. $Q P-P Q$ is compact and $z^{-}+z^{+}=2$ if and only if

$$
w=\left|h_{0}\right|^{2} e^{u+\tilde{v}}
$$

where $h_{0}$ is an outer function in $H^{2-}, \hbar_{0} / h_{0}=\bar{F}$ for some inner function $F$ and where $u$ and $v$ are real functions in $C$.

Proof. If $Q P-P Q$ is compact and $Z^{-}+Z^{+}=2$ then by Theorem 3 $H_{\bar{\phi}}$ is compact. Hence $\bar{\phi} \in H^{\infty}+C$. By a theorem of wolff [6] $\phi=\bar{F} e^{i(v-\tilde{u})}$ where $F$ is an inner function and where $u$ and $v$ are real functions in $C$. Put $g=e^{-\tilde{v}-u+i(v-\tilde{u})}$ then

$$
|g||h|^{2}=\bar{F} g h^{2} \geqslant 0 \quad \text { a.e. }
$$

If $h_{0}^{2}=g h^{2}$ then $h_{0}^{2} \in h^{2-}$ by a theorem of zygmund (see [4], p. 140). since $\left|h_{0}\right|^{2}=\bar{F} h_{0}^{2}, \bar{h}_{0} / h_{0}=\bar{F}$ and $w=\left|h_{0}\right|^{2} e^{u+\tilde{v}}$. Conversely if $|h|^{2}=\left|h_{0}\right|^{2} e^{u+\tilde{v}}$ then $\bar{\phi} \in H^{\infty}+C$. since $H_{\bar{\phi}}$ is compact, Theorem 3 implies the proposition.

Let $Z^{+/-}$be the closure of the projection of $Z^{+}$on $Z^{-}$then $Z^{-} \supset z^{+/-}$. Levinson and McKean ([5], pp. 103-105) showed that $Z^{-} \neq z^{+/-}$if and only if $\phi=\bar{F} G$ for some inner functions $F$ and $G$.

PROPOSITION 6. QP - PQ has finite ronk $2 n$ if and only if $\phi=\bar{F} G$ where $F$ is an inner function and $G$ is a finite Blashke product of degree $n$.

Proof. If $Q P-P Q$ has finite rank $2 n$ then by Theorem $4 H_{\bar{\phi}}$ has finite rank $n$. Then ker $H_{\bar{\phi}} \neq\{0\}$ and so $\operatorname{ker} H_{\bar{\phi}}=G H^{2}$ for some inner function $G$ by Beurling's theorem. Hence $\phi=\bar{F} G$ for some inner 
function $F$. Since $\operatorname{rank} H_{\bar{\phi}}=\operatorname{codim} \operatorname{ker} H_{\bar{\phi}}=\operatorname{dim}\left(H^{2} \Theta G H^{2}\right), G$ is a finite Blashke product of degree $n$. Conversely if $\phi=\bar{F} G$ and $G$ is a finite Blashke product of degree $n$ then $H_{\bar{\phi}}$ has finite rank $n$. Theorem 4 implies $Q P-P Q$ has finite rank $2 n$.

Hayashi ([1], Theorem 2) showed that $Q P-2 \wedge P$ is compact if and only if $H_{\bar{\phi}}$ is compact. The proof shows that $Q P-Q \wedge P$ has finite rank $n$ if and only if $H_{\bar{\phi}}$ has finite rank $n$. Thus rank $(Q P-P Q)=2 \operatorname{rank}(Q P-Q \wedge P)$.

\section{References}

[1] E. Hayashi, "The spectral density of a strongly mixing stationary Gaussian process", Pacific J. Math., 96 (1981), 343-359.

[2] H. Helson and D. Sarason, "Past and future", Math. Scand., 21 (1967), $5-16$.

[3] H. Helson and G. Szegö, "A problem in prediction theory", Ann. Mat. Pura App Z., 51 (1960), 107-138.

[4] P. Koosis, "Introduction To $H_{p}$ Spaces", London Math. Soc. Lecture Note Ser., 40 (1980).

[5] N. Levinson and H.P. MCKean, "Weighted trigonometrical approximation on the line with application to the germ field of a stationary Gaussian noise", Acta. Math., 112 (1964), 99-143.

[6] T.H. Wolff, "Two algebras of bounded functions", Duke Math. J., 49 (1982), 321-328.

Department of Mathematics,

Faculty of Science,

(General Education)

Hokkaido University,

Sapporo 060, Japan. 